\title{
Effect of Hydrogen Radical Treatment and Synthesis of Sn-Catalyzed Silicon Nanowires at Various Growth Conditions
}

\author{
Hisashi Uchiyama, Minsung Jeon,* Yoshihiro Tomitsuka, Kazuya Maishigi, and Koichi Kamisako \\ Department of Electrical and Electronic Engineering, Tokyo University of Agriculture and technology, \\ 2-24-16 Nakacho, Koganei-shi, Tokyo 184-8588, Japan \\ (Received 2 March 2009; Accepted 13 April 2009; Published 13 June 2009)
}

\begin{abstract}
Tin-catalyzed silicon nanowires (SiNWs) are synthesized using the hydrogen radical-assisted deposition method. Numerous metal nanoparticles are fabricated after hydrogen radical pretreatment from metal thin film. Large quantities of SiNWs after formation of metal catalysts are synthesized at various growth conditions, such as varied nanoparticle sizes, growth times and temperatures. Their diameters and lengths depend on the growth time and size of nanoparticles. Voluminous whisker-like crystallized SiNWs are obtained at temperatures above $350^{\circ} \mathrm{C}$. [DOI: $10.1380 /$ ejssnt.2009.708]
\end{abstract}

Keywords: Hydrogen radicals; Silicon nanowires (SiNWs); Sn catalyst; FE-SEM

\section{INTRODUCTION}

Low-dimensional semiconducting nanostructures, such as nanotubes (NTs), nanowires (NWs), nanorods and nano-ribbons, are expected to an important role as both interconnect and functional units in the fabricating electronic, optoelectronics, electrochemical and electromechanical devices with nanoscale dimensions [1]. For fabricating semiconductor nanostructures, various synthesis methods, such as chemical vapor deposition (CVD), laser ablation, solution, oxide-assisted and template-assisted method [2-5], have been proposed by many research groups. In order to synthesize semiconducting nanostructures, moreover, metal nanoparticles are important because the nanostructures are grown via supersaturation of metal-catalyzed semiconductor source, which means wellknown vapor-liquid-solid (VLS) mechanism [6].

In recent, silicon nanowires (SiNWs) have attractive great interest due to their possibility as electronic and optic devices, and semiconducting properties [7, 8]. For synthesis of SiNWs, various metal catalysts, such as Au, $\mathrm{Ti}, \mathrm{In}, \mathrm{Ga}$ and $\mathrm{Al}$ [9-13], are used and suggested. In particular, Au and Ti nanoparticles are well used for growth of SiNWs. However, they form deep level impurities in silicon bulk. Therefore, to find useful metal catalysts is one of the key issues for synthesis of SiNWs. In our previous work, we suggested Sn catalyst, which has low eutectic point with Si [14], and successfully synthesized large quantities of SiNWs. Furthermore, we have evidenced the effectiveness of the hydrogen radical treatment for fabricating nanoparticles from metal thin films [13] and obtaining voluminous SiNWs.

In this work, therefore, we investigate the effect of hydrogen radical treatment for fabricating metal nanoparticles from Sn thin film and synthesizing SiNWs. In addition, Sn-catalyzed SiNWs are synthesized by the hydrogen radical-assisted deposition method [15] at various growth conditions, such as synthesis times and temperatures. The fabricated nanostructures are explored by field-emission scanning electron microscopy (FE-SEM) measurement.

*Corresponding author: joseph@cc.tuat.ac.jp

\section{EXPERIMENTAL}

For evaporating Sn thin film, a single side polished $\mathrm{Cz}$ (100) silicon substrate was used. The substrate was dipped in diluted $5 \%$ hydrofluoric (HF) acid to remove a native oxide layer formed on the substrate, and rinsed in deionized water. Subsequently, the substrate was located into the thermal evaporation equipment. Then, Sn thin film with approximately $40 \mathrm{~nm}$-thick was deposited onto the Si substrate by an evaporation sources. After metal film evaporation, the sample was immediately set into the experimental chamber and heated at $400^{\circ} \mathrm{C}$ with a pressure of $2 \times 10^{-5}$ Torr. To fabricate $\mathrm{Sn}$ nanoparticles as catalysts from $\mathrm{Sn}$ thin film, hydrogen $\left(\mathrm{H}_{2}\right)$ gas as carrier gas was introduced through $1 / 2$-inch trumpet-like quartz tube. The $\mathrm{H}_{2}$ gas was discharged by $2.45-\mathrm{GHz}$ microwave and then the discharged $\mathrm{H}_{2}$ gas, i.e., hydrogen radicals $(\mathrm{H})$, was irradiated onto the sample for 1 and $10 \mathrm{~min}$. After hydrogen radical treatment at varied treatment times, the morphological properties on the sample were observed by FE-SEM measurement. Furthermore, silane $\left(\mathrm{SiH}_{4}\right)$ gas as a $\mathrm{Si}$ source was introduced on the samples fabricated at varied hydrogen radical treatment times for synthesizing SiNWs. The $\mathrm{H}_{2}$ and $\mathrm{SiH}_{4}$ gas flow rates were 180 and 12 sccm, respectively. The SiNWs were synthesized for $20 \mathrm{~min}$ at $400^{\circ} \mathrm{C}$. Detailed conditions for hydrogen radical treatment and synthesis of SiNWs were summarized in Table I. After synthesis of SiNWs, their characteristics were also analyzed by FE-SEM observation.

\section{RESULTS AND DISCUSSION}

Figures 1(a) and (b) show the FE-SEM images of Sn nanoparticles fabricated on Si substrate after hydrogen radical treatment at varied treatment times: (a) 1 min and (b) $10 \mathrm{~min}$. As can be seen in these figures, voluminous spherical nanostructures were fabricated on the substrates after $\mathrm{H}$ treatment. Moreover, the sizes of nanoparticles were decreased with increasing $\mathrm{H}$ treatment time (see inset of Figs. 1(a) and (b)). When the $\mathrm{H}$ treatment time was $1 \mathrm{~min}$, the distribution of fabricated nanoparticles was dense and their sizes were almost same with approximately $60 \mathrm{~nm}$ in diameter (see Fig. 1(a)). On the other hand, when the $\mathrm{H}$ treatment time was increased, large nanoparticles with about $100 \mathrm{~nm}$ were fabricated 
TABLE I: Conditions for fabrication of metal nanoparticles from metal thin film and synthesis of silicon nanowires (SiNWs).

\begin{tabular}{lcc}
\hline \hline & H treatment & Synthesis of SiNWs \\
\hline Hydrogen $\left(\mathrm{H}_{2}\right)$ gas flow rate $[\mathrm{sccm}]$ & 100 & 180 \\
Silane $\left(\mathrm{SiH}_{4}\right)$ gas flow rate $[\mathrm{sccm}]$ & - & 12 \\
Microwave power $[\mathrm{W}]$ & 40 & 40 \\
Working pressure $[$ Torr] & 0.5 & 0.5 \\
Temperature $\left[{ }^{\circ} \mathrm{C}\right]$ & 400 & 400 \\
Time $[\mathrm{min}]$ & 1,10 & 20 \\
\hline \hline
\end{tabular}
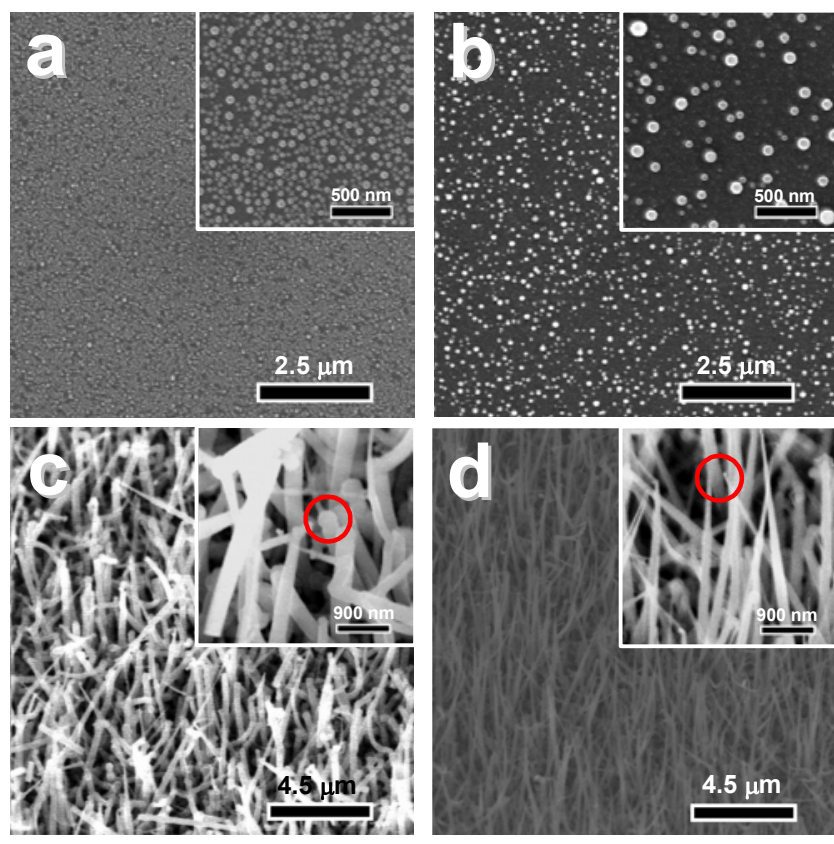

FIG. 1: (a) and (b) shows the FE-SEM images of Sn nanoparticles as catalyst fabricated for various hydrogen radical treatment times: (a) $1 \mathrm{~min}$ and (b) $10 \mathrm{~min}$. (c) and (d) represent synthesized SiNWs after fabrication of Sn nanoparticles as shown in (a) and (b). The fabrication temperature and time were set to $400^{\circ} \mathrm{C}$ and $20 \mathrm{~min}$, respectively.

as shown in Fig. 1(b). Furthermore, very small nanoparticles with approximately $10 \mathrm{~nm}$ were also observed as shown in inset of Fig. 1(b). Here, the formation of large nanoparticles might be the aggregation of small nanoparticles for increasing $\mathrm{H}$ treatment time. From these results, we found that the size of nanoparticles depends strongly on the $\mathrm{H}$ treatment time and it can be controlled by the $\mathrm{H}$ treatment conditions.

Subsequently, for synthesizing SiNWs, a $\mathrm{SiH}_{4}$ gas was introduced on the fabricated samples as show in Figs. 1(a) and (b). The growth conditions were detailed in Table I. Figures 1(c) and (d) show the FE-SEM images of the synthesized SiNWs using varied Sn nanoparticles size. Large quantities of SiNWs were synthesized on the both substrates as shown in Figs. 1(c) and (d). The structure of SiNWs was whisker-like. When the size of nanoparticles was large (see Fig 1(a)), the synthesized SiNWs were approximately $400 \mathrm{~nm}$ in diameter and their lengths were extended to $3 ? \mathrm{~m}$. For SiNWs grown by small nanoparticles

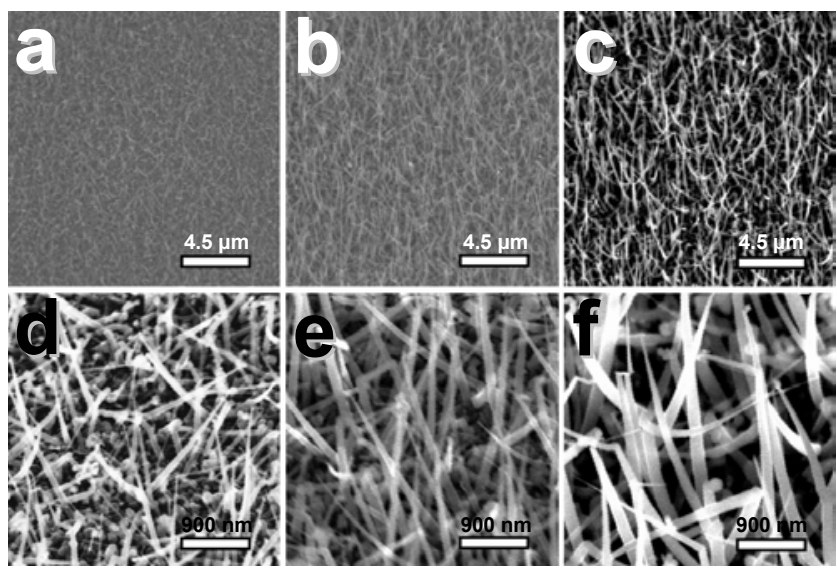

FIG. 2: FE-SEM images of SiNWs synthesized for various growth times: (a, d) 5 min, (b, e) $10 \mathrm{~min},(\mathrm{c}, \mathrm{f}) 15 \mathrm{~min}$. Bottom side represents the high-magnification FE-SEM images as shown in (a), (b), and (c).

(see Fig. 1(b)), thin and long SiNWs were synthesized. It indicates that the SiNWs depend on the nanoparticlesf sizes.

Moreover, the Sn catalysts are revealed on the top of SiNWs (see red circles inset of Fig. 1(c, d)). It means that the SiNWs were synthesized via VLS mechanism [6]. However, the diameters of SiNWs were thicker than metal nanoparticles. It might be the effect of co-occurred axial growth via metal-catalyzed VLS mechanism and radial growth via vapor-solid mechanism.

To investigate the dependence of Sn-catalyzed SiNWs growth time, the synthesis was changed from 5 min to 20 min. After synthesis of SiNWs, their structural properties were observed by FE-SEM measurement. As-mentioned above, before synthesizing SiNWs, hydrogen radical treatment was performed on the Sn-coated substrates for $1 \mathrm{~min}$ at $400^{\circ} \mathrm{C}$. Then, the SiNWs were synthesized for various growth times. Figure 2 shows the morphological properties of SiNWs synthesized for (a, d) 5min, (b, e) 10min and (c, f) $15 \mathrm{~min}$. Voluminous SiNWs were whisker-likely synthesized. When the synthesis time was short as 5 min, SiNWs with random growth direction and small quantity were obtained as shown in Figs. 2(a) and (d). On the other hand, when the growth time was above $10 \mathrm{~min}$, their growth directions were almost perpendicularly revealed against sample substrate. Moreover, their diameters became thicker from about $100 \mathrm{~nm}$ to $\sim 400 \mathrm{~nm}$ (see Fig. 2(e) and (f) and inset of Fig. 1(c)). In contrast with 


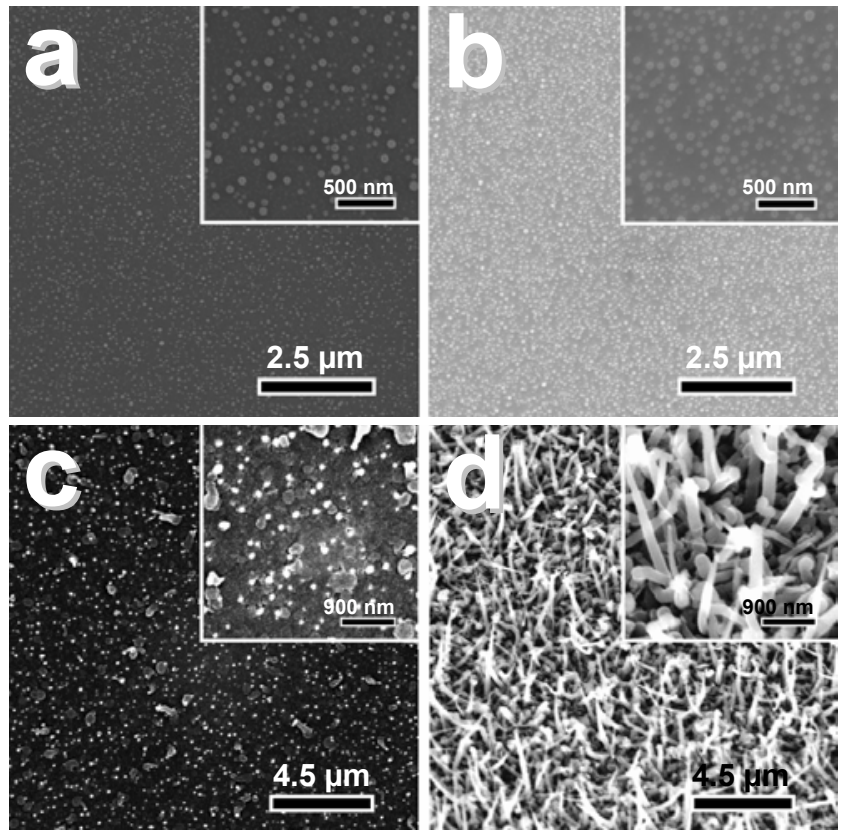

FIG. 3: FE-SEM images of the fabricated nanoparticles and synthesized SiNWs at various formation temperatures: (a, c) $300^{\circ} \mathrm{C}$ and $(\mathrm{b}, \mathrm{d}) 350^{\circ} \mathrm{C}$. The hydrogen radical treatment and growth time of SiNWs were set to $1 \mathrm{~min}$ and $20 \mathrm{~min}$, respectively.

the diameters, their lengths were almost same with approximately $3 \mu \mathrm{m}$. These results may be caused by the above-mentioned metal catalyst size. Moreover, the thickening SiNWs in diameters may result by the radial growth mechanism with increasing growth time. From these results, we confirm that the diameter and length of SiNWs depend on the growth time and nanoparticle size, respectively.

In addition, SiNWs were synthesized at various temperatures from 300 to $400^{\circ} \mathrm{C}$ and their characteristics were explored. Before synthesis of SiNWs, hydrogen radical treatment was performed for $1 \mathrm{~min}$ at various temperatures. Subsequently, a $\mathrm{SiH}_{4}$ gas as silicon source was fed for $20 \mathrm{~min}$ on the samples to grow SiNWs. Other growth conditions were same in Table I. The surface morphologies before and after synthesis of SiNWs were analyzed by FE-SEM observation. Figure 3 shows the FE-SEM images of sample surface after $\mathrm{H}$ treatment and synthesis of SiNWs at various temperatures: (a, c) $300^{\circ} \mathrm{C}$ and (b, d) $350^{\circ} \mathrm{C}$. As can be seen in Figs. 3(a), (b) and Fig. 1(a), the quantities of nanoparticles were increased with increasing $\mathrm{H}$ treatment temperature. After synthesis of SiNWs, voluminous whisker-like SiNWs were observed at higher temperatures above $350^{\circ} \mathrm{C}$ (see Fig. 3(d) and Fig. 1(c)). It denotes that the growth temperature for synthesizing wire-like SiNWs must be above $300^{\circ} \mathrm{C}$. The temperature dependency was well described in our previouswork [16].

\section{CONCLUSIONS}

Sn-catalyzed SiNWs were synthesized by the hydrogen radical-assisted deposition method including hydrogen radical pretreatment. The effect of hydrogen radical treatment times, growth time and temperature for synthesizing SiNWs was examined. In this experiment, we found that the size of nanoparticles and growth time dominated the diameter and length of SiNWs, respectively. Furthermore, the growth temperature for synthesizing wire-like SiNWs must be higher than $300^{\circ} \mathrm{C}$.

\section{Acknowledgments}

The authors acknowledge Prof. Minoru Aoyagi of Nippon Institute of Technology for expert experimental assistance.
[1] Z. L. Wang, Nanowires and Nanobelts: Materials, Properties and Devices (Kluwer Academic Publishers, 2003).

[2] A. M. Morales and C. M. Lieber, Science 279, 208 (1998).

[3] J. D. Holmes, K. P. Johnston, R. C. Doty, and B. A. Kogel, Science 287, 1471 (2000).

[4] N. Wang, Y. H. Tang, Y. F. Zhang, C. S. Lee, I. Bello, and S. T. Lee, Chem. Phys. Lett. 299, 237 (1999).

[5] T. E. Bogart, S. Dey, K. K. Lew, S. E. Mohney, and J. M. Redwing, Adv. Mater. 17, 114 (2005).

[6] R. S. Wagner and W. C. Ellis, Appl. Phys. Lett. 4, 89 (1964).

[7] X. B. Zeng, X. B. Liao, B. Wang, S. T. Dai, Y. Y. Xu, X. B. Xiang, Z. H. Hu, H. W. Diao, and G. L. Kong, J. Cryst. Growth 265, 94 (2004).

[8] D. B. Migas, J. Appl. Phys. 102, 064301 (2007).

[9] J. Westwater, D. P. Gosain, S. Tomiya, S. Usui, and H. Ruda, J. Vac. Sci. Technol. B 15, 554 (1997).
[10] T. I. Kamins, R. S. Williams, Y. Chen, Y. L. Chang, and Y. A. Chang, Appl. Phys. Lett. 76, 562 (2000).

[11] M. S. Jeon, Y. Tomitsuka, M. Aoyagi, and K. Kamisako, Jpn. J. Appl. Phys. 48, 015002 (2009).

[12] M. S. Jeon, Y. Tomitsuka, and K. Kamisako, J. Ind. Eng. Chem. 14, 836 (2008).

[13] M. S. Jeon and K. Kamisako, J. Alloys compd. 476, 84 (2009).

[14] M. S. Jeon, H. Uchiyama, and K. Kamisako, Mater. Lett. 63, 246 (2009).

[15] M. S. Jeon and K. Kamisako, Mater. Lett. 62, 3903 (2008).

[16] M. S. Jeon, Y. Tomitsuka, K. Maishigi, H. Uchiyama, M. Aoyagi, and K. Kamisako, IEICE Electron. Express 5, 586 (2008). 